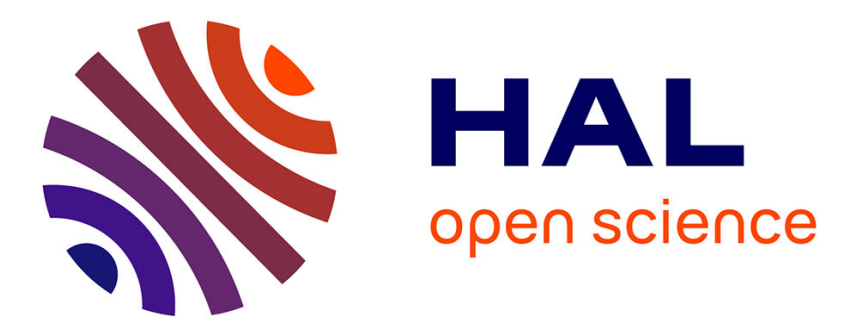

\title{
A Possibilistic Framework for Identifying the Performance to be Improved in the Imprecise Context of Preliminary Design Stage
}

Diadie Sow, Abdelhak Imoussaten, Pierre Couturier, Jacky Montmain

\section{- To cite this version:}

Diadie Sow, Abdelhak Imoussaten, Pierre Couturier, Jacky Montmain. A Possibilistic Framework for Identifying the Performance to be Improved in the Imprecise Context of Preliminary Design Stage. CIVEMSA 2017, Jun 2017, Annecy, France. 10.1109/CIVEMSA.2017.7995301 . hal-01556487

\author{
HAL Id: hal-01556487 \\ https://hal.science/hal-01556487
}

Submitted on 5 Jul 2017

HAL is a multi-disciplinary open access archive for the deposit and dissemination of scientific research documents, whether they are published or not. The documents may come from teaching and research institutions in France or abroad, or from public or private research centers.
L'archive ouverte pluridisciplinaire HAL, est destinée au dépôt et à la diffusion de documents scientifiques de niveau recherche, publiés ou non, émanant des établissements d'enseignement et de recherche français ou étrangers, des laboratoires publics ou privés. 


\title{
A Possibilistic Framework for Identifying the Performance to be Improved in the Imprecise Context of Preliminary Design Stage
}

\author{
Diadie Sow, Abdelhak Imoussaten, Pierre Couturier and Jacky Montmain \\ École des Mines d'Alès - LGI2P \\ F30035 Nîmes cedex 5, France \\ Email: firstname.name@mines-ales.fr
}

\begin{abstract}
In a highly competitive and unstable environment, manufacturers must constantly improve their products to remain competitive and satisfy their customers while minimizing incurred costs and risk taking. At the early stages of (re-) engineering, performances forecasting of new product is complicated. Indeed, the impacts of any characteristic change on the product performance are not precisely known. Decisionmakers must thus identify the performances to be improved while limiting the engineering efforts spent on innovative upgrades. Although some theoretical worth indexes have been proposed in the multiple criteria literature to estimate the expectable gains when improving changes are planned, they generally rely on non-realistic assumptions on the achievability of the expected improvements. Based on multi-criteria decision analysis techniques and uncertainty theory, this paper proposes an extension of the worth index concept when the likelihood of the expected improvements is not precisely known as it is the case at the preliminary stages of design activities.
\end{abstract}

Keywords: Performance Evaluation; Imprecise Assessment; Multiple criteria Decision Analysis; Possibility Theory

\section{INTRODUCTION}

The question addressed in this paper is: how to identify the characteristics of a product which are the most likely profitable ones to be improved when improvements's consequences are only imprecisely known and assessment of products is multidimensional? This problem is posed to manufacturers who must, in a highly competitive and unstable environment, constantly improve their products to remain competitive and satisfy their customers while minimizing incurred costs and risk taking. Many constraints must be taken into account when designing or when improving a product [1][2]. The number of components, functions, and interactions within complex products is becoming so substantial that responding to the multiple needs of customers, and moreover at lower cost is anything but obvious [3][4]. Such a challenge requires large forecasting capacities in order engineers to be able to produce challenging but achievable goals. The problem to be solved for the industrial manufacturer is how to design new products or improve products according to customers's requirements at the limit of what is technically feasible as he is aware of his available enterprise-level skills. At the early stages of design activities, performance forecasting of new products is complicated by the only qualitative characteristic of available knowledge. Indeed, at these stages, engineers cannot precisely estimate the impacts of design decisions; generally, they can only estimate them in a purely qualitative manner. Defining achievable targets is thus a risky and not deterministic process.

That is why, decisional strategies have to be set out to define, compare and select potential improvement actions with respect to stakeholder's needs. The relationships existing between the multiple performances of the product significantly complicate the choice. Hence, adequate mathematical models are required to deal with multiple assessment criteria in uncertain environment [5] [6].

It is the purpose of multi-criteria decision analysis (MCDA), to compare solutions according to the Decision-maker's (DM) preferences. An aggregation operator may be identified to assess all the alternatives w.r.t the criteria and the DM's preferences. Aggregation operators are often limited to simple linear operators such as weighted arithmetic means. However, such aggregation operators require independence between criteria, which is a rather drastic hypothesis [7]. Indeed, in practice, preferential interactions may make counter-intuitive the overall result of elementary improvements w.r.t to specific criteria. The family of fuzzy integrals like the Choquet integral (quantitative framework) and the Sugeno integral (qualitative framework) allows modelling both the preferential interactions between performances and their relative importance in the decisional strategy of the DM through a fuzzy measure[8]. Recently, some works exploit the preference model of the multiple criteria decision framework, in particular the Choquet integral, to estimate the expected improvements of an alternative. The authors in [9] [10] [11] associate to any coalition of criteria an index named worth index that measures for any alternative, the mean of all the possible expected improvements w.r.t a subset of criteria. These models assume that all the improvements are equiprobable whatever their magnitude. This assumption can clearly be challenged in practice. In [12] an extension of the worth index in a probability framework is proposed. Improvements magnitude obeys probability laws that are learned from data. However, such a framework relies upon the data availability which is not often the case in the context of this paper i.e. the preliminary stages of a product re- 
engineering. For example, a mobile robot manufacturer may know that tracked robot products are well adapted to loose grounds but their speed, reliability or energy consumption performances depend on many other technical choices, making their assessment both imprecise and uncertain until the robot is built. To represent performance in such a context subject to imprecision and uncertainty, possibility theory is an adequate framework. Therefore, an extension of the concept of worth index into a possibilistic framework is proposed here. For instance, in the robot example, this paper answers the following question: which performance features of an existing mobile robot product should be improved in order to satisfy new customer's needs without spending too much time and money? The paper will be divided as follows: Section 2 briefly reminds some essential MAUT notions to define the worth index. Section 3 gives possibility distribution definitions and some necessary reminders to understand our proposal. Section 4 introduces and discusses two possibilistic representations for the worth index. Finally, section 5 presents a case study of our approach to the re-engineering problem of a mobile robot.

\section{ASSESSMENT AND IMPROVEMENT IN A MULTI} CRITERIA FRAMEWORK: REMINDING AND NOTATIONS

Defining an improvement within the multi-criteria performance context poses a number of problems. First of all, it must be possible to compare any two of the situations described by their performances on all characteristics. Without this partial order, no sense can be assigned to the concept of improvement. Multi-criteria decision analysis can thus consist of reducing dimensionality in order to facilitate analysis. It is generally recognized that humans lose their ability to make rational decisions once more than 3 to 5 criteria are involved.

\section{A. Multi Attribute Utility Theory (MAUT)}

Let us denote by $N=\{1,2, \ldots, n\}$ a set of attributes where the $i^{t h}$ attribute takes its values in a set denoted $X_{i}$.

The aim of MAUT is to model the preferences of the DM, represented as a binary relation $\preceq$ on $X=\prod_{i \in N} X_{i}$ by means of an overall utility function $U: X \rightarrow \mathbb{R}$ such that $x \preceq y \Leftrightarrow U(x) \leq U(y), \forall x, y \in X$. This global utility can be seen as a numerical representation of the preference relation $\preceq$ on $X$ and captures a model of the expertise of the DM. As far as the overall utility function is considered, the most frequently encountered model is the additive value model. The transitive decomposable model of Krantz et al. [13] generalizes this representation as $U(x)=$ $H\left(u_{1}\left(x_{1}\right), u_{2}\left(x_{2}\right), \ldots, u_{n}\left(x_{n}\right)\right), \forall x=\left(x_{1}, x_{2}, \ldots, x_{n}\right) \in X$, where $\forall i \in N$, the function $u_{i}: X_{i} \rightarrow \mathbb{R}_{+}$is the utility function, it can be interpreted as a measure of the satisfaction of the value $x_{i}$ for the DM and $H: \mathbb{R}_{+}^{n} \rightarrow \mathbb{R}_{+}$, non-decreasing in its arguments, is the aggregation function [14].

Although the additive form is the most widespread because of its simplicity and its intuitive interpretation, it relies on strong assumptions such as the independence and the weakly separability of the criteria [15]. In practice attributes may interact with each other and preferential interactions are to be considered.

To overcome this problem, the Choquet integral is an interesting candidate for operator $H$. Indeed, it generalizes several classical operators, such as the weighted sum of the additive model or the ordered weighted sum (OWA) [7]. In addition, it allows modelling interactions between attributes. In the following, we propose a brief reminder about fuzzy measures and the Choquet integral.

\section{B. The Choquet Integral}

Definition II.1. A fuzzy measure $\mu$ on $N$ is a set function: $\mu: 2^{N} \rightarrow[0,1]$ that satisfies the following conditions:

- $\mu(\emptyset)=0 ; \mu(N)=1$;

- $\mu$ is monotonic non decreasing for inclusion, i.e., for any $A, B \subseteq N, A \subseteq B \Rightarrow \mu(A) \leq \mu(B)$

In the context of multi-criteria decision analysis, $\mu(I)$ represents the importance coefficient of the coalition of criteria $I \subseteq N$. Note that the monotonicity requirement of a fuzzy measure is weaker than the additivity requirement in usual cases.

Definition II.2. In the context of multi-criteria decision analysis, the evaluation of the Choquet integral of an alternative $x=\left(x_{1}, x_{2}, \ldots, x_{n}\right) \in \mathbb{R}_{+}^{n}$ with respect to $\mu$ denoted by $C_{\mu}(x)$ is defined by:

$$
C_{\mu}(x)=\sum_{i=1}^{n}\left(x_{\sigma(i)}-x_{\sigma(i-1)}\right) \mu\left(A_{\sigma(i)}\right)
$$

where $x_{\sigma(0)}=0 \leq x_{\sigma(1)} \leq x_{\sigma(2)} \leq \cdots \leq x_{\sigma(n)} ; A_{\sigma(i)}=$ $\{\sigma(i), \sigma(i+1), \ldots \sigma(n)\}$ where $\sigma$ is a permutation on $N$.

To identify the fuzzy measure $\mu$ associated to the Choquet integral, some methods have been proposed in the literature but are out the scope of this paper. We especially use the MACBETH extension to Choquet integral proposed in [8]. We adopt the following notation in this paper: for $I \subseteq N$, $\left(y_{I}, z_{-I}\right)$ is the vector $x \in X$ such that $x_{i}=y_{i}$ if $i \in I$ and $x_{i}=z_{i}$ if $i \in N \backslash I$.

\section{Worth Index}

In [9] it is proposed an index for determining the subset of criteria on which an alternative should be first improved in order to raise its overall score to the greatest extent possible. In [10] the author introduces the worth index denoted by $w_{H}\left(x^{0}, I\right)$, for the aggregation function $H$ and initial performances $x^{0} \in \mathbb{R}_{+}^{n}$ that assesses the possible gain the coalition of criteria $I \subset N$ could bring to the overall performance as it is modelled by the $H$-aggregation of the elementary performances. For any $I \subseteq N, w_{H}\left(x^{0}, I\right)$ is the worth for alternative $x^{0}$ to be improved on the subset of criteria $I$, subject to the evaluation function $H$. In this paper, only the case $H=C_{\mu}$ will be considered. More precisely, the worth index $w_{C_{\mu}}\left(x^{0}, I\right)$ is defined by:

$$
\begin{aligned}
& w_{C_{\mu}}\left(x^{0}, I\right)= \\
& \quad \int_{0}^{1}\left[C_{\mu}\left((1-\lambda) x_{I}^{0}+\lambda 1_{I}, x_{-I}^{0}\right)-C_{\mu}\left(x^{0}\right)\right] d \lambda
\end{aligned}
$$


The choice to integrate in formula (2) along the hyper bisector ensures a homogeneous improvement of all the criteria in $I$. The main drawback there is in the two approaches proposed in [9][10] is that all the performance profiles are supposed to be equally probable which is evidently not the case in practice (large improvements are less probable than tiny ones). In [12] the authors propose an extension of the worth index in a probabilistic framework where, a probability value is assigned to each improvement $x \in X$ starting from $x^{0}$ :

$$
\begin{aligned}
& w_{C_{\mu}}\left(x^{0}, I\right)= \\
& \quad \int_{\left[x^{0} \uparrow\right]} p_{I}\left(x_{I}, x_{-I}^{0}\right)\left[C_{\mu}\left(x_{I}, x_{-I}^{0}\right)-C_{\mu}\left(x^{0}\right)\right] d x_{I}
\end{aligned}
$$

where $\left[x^{0} \uparrow\right]$ is the set of alternatives that improves $x_{0}$ over $I, p_{I}(x)$ expresses the probability to obtain the alternative $x$ and $C_{\mu}$ is the Choquet integral w.r.t the fuzzy measure $\mu$.

In practical cases, identifying the probability distributions $p_{I}\left(x_{I}, x_{-I}^{0}\right)$ depends on the availability of a learning data base [12]. However, in preliminary design stages, experts are mostly able to provide qualitative estimations of these distributions. In this case, estimating the distributions is no more based on a statistic learning stage. In order to integrate this qualitative expert knowledge in the computation of the worth index, this paper proposes a new model for the worth index in a possibilistic framework.

\section{POSSIBILITY THEORY}

Possibility theory is an appropriate framework to represent imprecise and uncertain information [16]. It is based on a possibility distribution which is a membership function to which two fuzzy measures are associated named possibility and necessity measures.

Definition III.1. A possibility distribution $\pi$ assigns to each element $\omega$ in a set $\Omega$ of alternatives a degree of possibility $\pi(\omega) \in[0,1]$ of being the correct description of a state of the world.

- $\pi(\omega)=0$ means that $\omega$ is impossible.

- $\pi(\omega)=1$ means that $\omega$ is totally possible.

- $[b, c]=\{\omega / \pi(\omega)=1\}$ is the kernel of $\pi$.

- $] a, d[=\{\omega / \pi(\omega)>0\}$ is the support of $\pi$.

- to $\pi$ are associated two fuzzy measures $\Pi$ and $N$.

- $\forall A \subseteq \Omega, \Pi(A)=\max _{\omega \in A} \pi(\omega)$.

- $\forall A \subseteq \Omega, N(A)=\inf _{\omega \notin A}(1-\pi(\omega))$.

- $\forall A \subseteq \Omega, N(A)=1-1-\Pi\left(A^{c}\right)$.

By analogy with the mathematical expectation of a random variable, the expectation associated to a possibility distribution is also defined in [17].

Definition III.2. Given a possibility distribution $\pi$, two cumulative distributions can be associated to it through $\Pi$ and $N$ and are respectively named upper distribution $F_{*}$ and lower distribution $F^{*}$ defined by: $F^{*}(a)=\Pi((-\infty, a])=$ $\sup \{\pi(\omega), \omega \leq a\}$ and $F_{*}(a)=N((-\infty, a])=\inf \{1-$ $\pi(\omega), \omega>a\}$.
The mathematical expectation associated to $\pi$ is imprecise and is then defined in [17] by the mean interval:

$$
E(\pi)=\left[E_{*}(\pi), E^{*}(\pi)\right],
$$

where $E_{*}(\pi)=\int_{-\infty}^{+\infty} x d F^{*}(x)$ and $E^{*}(\pi)=\int_{-\infty}^{+\infty} x d F_{*}(x)$. Roughly speaking and to make the analogy with probabilities, the 'saverage's value of $\pi$ would be the mean interval $\left[E_{*}(\pi), E^{*}(\pi)\right]$. A 'smeasure of dispersion's can also be introduced: $\Delta(\pi)=E^{*}(\pi)-E_{*}(\pi)$. It may be useful to define a location indicator which allows synthesising the possibility distribution into a unique precise representative value in order to facilitate comparing possibility distributions [18].

The intuitive idea beyond this work is that a possibility distribution can be a priori attached by experts to the expected values of each $x_{i}$. Then, the expected performances w.r.t criterion $i$ are modelled by a possibility distribution $\pi_{i}$ over the value range of the $x_{i}$ 's. As a consequence, the aggregation step that computes the aggregated performance from the elementary ones must be extended to the case of imprecise and uncertain inputs. In other words, the aggregation process must be extended when inputs are possibility distributions [18] [19].

\section{A. Aggregating Possibility Distributions with a Choquet Inte- gral}

Let us consider $n$ elementary possibility distributions $\left(\pi_{1}, \pi_{2}, \ldots, \pi_{n}\right)$ where $\pi_{i}$ is the possibility distribution related to the $i^{\text {th }}$ attribute. The aggregated possibility distribution with respect to the Choquet integral $\pi_{C_{\mu}}=C_{\mu}\left(\pi_{1}, \pi_{2}, \ldots, \pi_{n}\right)$ can be computed using the extension principle of Zadeh [20] which states that: $\forall z \in[0,1]$

$$
\pi_{C_{\mu}}(z)=\sup _{\substack{y=\left(y_{1}, \ldots, y_{n}\right) \in[0,1]^{n} \backslash \\ z=C_{\mu}(y)}} \min \left(\pi_{1}\left(y_{1}\right), \ldots, \pi_{n}\left(y_{n}\right)\right)
$$

The direct computation of the aggregated possibility distribution by this formula may be extremely time consuming, but in the case of the Choquet integral, it is proposed in [21] a more practical way to compute the aggregated distribution $\pi_{C_{\mu}}$ based on the hyperplan-linearity of the Choquet integral (the Choquet integral in (eq. (1)) behaves as a weighted mean in each hyperplan $\left.H_{\sigma} \backslash y_{\sigma(1)} \leq y_{\sigma(2)} \leq \ldots y_{\sigma(n)}\right)$. The computation only needs to be processed on a finite set of particular points.

\section{POSSIBILISTIC EXTENSION OF THE WORTH INDEX}

For sake of simplicity, we will denote a vector of performances associated to an alternative $x$ as $\left(x_{1}, \ldots, x_{n}\right) \in[0,1]^{n}$ instead of $\left.\left(u_{(} x_{1}\right), \ldots, u_{n}\left(x_{n}\right)\right)$. Let $x^{0} \in[0,1]^{n}$ the actual performances from which the improvement is envisaged. For each $i \in N$, a possibility distribution $\pi_{i}$ describes which performances are plausible and less plausible on the $i^{t h}$ attribute. We assume that experts are able to provide the possibility distributions $\pi_{i}$ over the value range of each $x_{i}$ 's. From their 
experience they know the minimal and maximal performance they have already met providing the kernel limits of each $\pi_{i}$. The value range of the support with a degree of possibility lower than 1 corresponds to possible values from the expert points of view although he has never met them before. For the sake of simplicity, only trapezoidal distributions are considered in this paper.

The issue is to identify on which attributes the initial state of performances should be improved in order to raise its overall performance to the greatest extent. The fewer these attributes are, the lower the cost of the increase. The worth index allows assessing the average expected gain of each coalition of improved performance attributes, and therefore allows selecting the coalition that provides the most relevant ratio cost/benefit. Possibility distributions will enlighten the DM on what is plausible and less plausible beyond $x^{0}$. Improving criteria in the coalition $I$ means that only performances related to criteria $I$ are improved whereas performances in $N \backslash I$ remain equal to $x_{0}^{i}$. For $i \in I$, only performances higher or equal to $x_{i}^{0}$ are possible. In other words the initial distributions $\pi_{i}$ are revised such as:

- for $i \in I, \pi_{i / x_{i}^{0}}\left(x_{i}\right)=0$ for any $x_{i} \leq x_{i}^{0}$, else $\pi_{i / x_{i}^{0}}\left(x_{i}\right)=\pi_{i}\left(x_{i}\right)$ (i.e., $\left.\pi_{i / x_{i}^{0}}=\pi_{i} \wedge 1_{\left[x_{0}, 1\right]}\right)$;

- for $i \in N \backslash I, \pi_{i / x_{i}^{0}}\left(x_{i}\right)=1$ if $x_{i}=x_{i}^{0}$, 0 elsewhere.

Two different approaches based on a possibilistic model of the worth index are now introduced to formalize the problem. The main advantage of the first one is that it relies solely on the descriptors of the possibility distributions (namely $E_{*}$ and $\left.E^{*}\right)$ and is thus easy-of-use but there is a loss of information content w.r.t the genuine knowledge the experts provide with the $\pi_{i}$ 's. The second one deals with the possibility distributions themselves: there is no loss of information but the calculations are heavier.

\section{A. Worth index based on the moments of the possibility} distributions

In this first approach a possibility distribution $\pi$ is synthesized by its lower and upper expected values $E_{*}(\pi)$ and $E^{*}(\pi)$. As consequence the worth index will be seen as an imprecise quantity and modeled by the interval:

$$
w_{C_{\mu}}\left(x_{0}, I\right)=\left[w_{* C_{\mu}}\left(x_{0}, I\right), w_{C_{\mu}}^{*}\left(x_{0}, I\right)\right]
$$

For $I \subseteq N$, the worth index $w_{C_{\mu}}\left(x_{0}, I\right)$ for a given fuzzy measure $\mu$ is defined with:

$$
\begin{gathered}
w_{* C_{\mu}}\left(x_{0}, I\right)=C_{\mu}\left(E_{*}\left(\pi_{I / x_{I}^{0}}\right), x_{-I}^{0}\right)-C_{\mu}\left(x^{0}\right) \\
w_{C_{\mu}}^{*}\left(x_{0}, I\right)=C_{\mu}\left(E^{*}\left(\pi_{I / x_{I}^{0}}\right), x_{-I}^{0}\right)-C_{\mu}\left(x^{0}\right)
\end{gathered}
$$

where $E_{*}\left(\pi_{I / x_{I}^{0}}\right)$ (resp. $E^{*}\left(\pi_{I / x_{I}^{0}}\right)$ ) denotes the vector of the $E_{*}\left(\pi_{i / x_{i}^{0}}\right)$ 's (resp. $E^{*}\left(\pi_{i / x_{i}^{0}}\right)$ 's) for $i$ in $I$.

Considering that experts can only provide simple distributions such as trapezoidal ones, we can compute for each $i$ in I:

- $E_{*}\left(\pi_{i / x_{i}^{0}}\right)=\int_{-\infty}^{+\infty} x_{i} d F_{i / x_{i}^{0}}^{*}\left(x_{i}\right)$, with $d F_{i / x_{i}^{0}}^{*}\left(x_{i}\right)=$ $\delta\left(x_{i}-x_{i}^{0}\right)$ the Dirac distribution at $x_{i}^{0}$, i.e., $\delta\left(x_{i}-x_{i}^{0}\right)=0$ if $x_{i} \neq x_{i}^{0}$ and $\delta(0)=+\infty$. Because $\int_{-\infty}^{+\infty} \delta\left(x_{i}-\right.$ $\left.x_{i}^{0}\right) d x_{i}=1$ and $f: x \mapsto x$ is a continuous function, $\int_{-\infty}^{+\infty} x_{i} \delta\left(x_{i}-x_{i}^{0}\right) d x_{i}=x_{i}^{0}$. Therefore $E_{*}\left(\pi_{i / x_{i}^{0}}\right)=x_{i}^{0}$

- $E^{*}\left(\pi_{i / x_{i}^{0}}\right)=\int_{-\infty}^{+\infty} x_{i} d F_{*_{i / x_{i}^{0}}}\left(x_{i}\right)=\int_{-\infty}^{+\infty} x_{i} d F_{* i}\left(x_{i}\right)$. In case of trapezoidal distributions $\int_{-\infty}^{+\infty} x_{i} d F_{* i}\left(x_{i}\right)=$ $\frac{d_{i}+c_{i}}{2}$ since $d F_{i}^{*}$ is a uniform probability law over $\left[c_{i}, d_{i}\right]$, where $\left[a_{i}, d_{i}\right]$ and $\left[b_{i}, c_{i}\right]$ are respectively the support and the kernel of the distribution $\pi_{i / x_{i}^{0}}$ for $i$ in $I$.

The interval $\left[E_{*}\left(\pi_{i / x_{i}^{0}}\right) ; E^{*}\left(\pi_{i / x_{i}^{0}}\right)\right]$ provides the expected mean value of $x_{i}$ when improvements on criterion $i$ in $I$ are performed. Thus, the expected mean gain for each criterion $i$ in $I$ is the interval $\left[0, \frac{d_{i}+c_{i}}{2}-x_{i}^{0}\right]$. Finally, the worth index is achieved with formulas (5) and (6). Note that other moments synthesizing possibility distributions can be found in [22].

\section{B. Worth index based on uncertainty propagation}

In this second approach, we propose to deal with the full information content of the possibility distribution. Let us now consider the information conveyed by the choice: starting from $x^{0}$ and improving only performances of attributes in $I$. The value range of the $x_{i}$ 's when improvements of the criteria in $I$ are envisaged, is described by the possibility distributions $\pi_{i / x_{i}^{0}}$ as proposed in the preceding subsection. The idea in this second approach is to propagate uncertainty about attributes performances through the Choquet integral to the aggregated distribution in order to compute the worth index. The computation is achieved using the Zadeh's extension principle and it gives: $\forall z \in[0 ; 1]$,

$$
\pi_{C_{\mu} / x^{0}, I}(z)=\sup _{\substack{y_{I} \in\left[x_{I}^{0}, 1_{I}\right], y_{-I}=x_{-I}^{0}: \\ z=C_{\mu}(y)}} \min \left(\pi_{I / x_{I}^{0}}\left(y_{I}\right)\right)
$$

We can now compute $E_{*}\left(\pi_{C_{\mu} / x^{0}, I}\right)$ and $E^{*}\left(\pi_{C_{\mu} / x^{0}, I}\right)$ and define the possibilistic worth index as the imprecise quantity: $w_{C_{\mu}}\left(x^{0}, I\right)=\left[w_{* C_{\mu}}\left(x_{0}, I\right), w_{C_{\mu}}^{*}\left(x_{0}, I\right)\right]$

where:

$$
w_{* C_{\mu}}\left(x_{0}, I\right)=E_{*}\left(\pi_{C_{\mu} / x^{0}, I}\right)-C_{\mu}\left(x^{0}\right)=0
$$

(by construction of the $\pi_{i / x_{i}^{0}}$ distributions)

$$
w_{C_{\mu}}^{*}\left(x_{0}, I\right)=E^{*}\left(\pi_{C_{\mu} / x^{0}, I}\right)-C_{\mu}\left(x^{0}\right)
$$

This worth index formulation should not be confused with the proposal in subsection A. Indeed, in the general case, $C_{\mu}\left(E_{*}\left(\pi_{1}\right), \ldots E_{*}\left(\pi_{n}\right)\right) \neq E_{*}\left(C_{\mu}\left(\pi_{1}, \ldots, \pi_{n}\right)\right)$ (see[18] for more details). The main advantage of the proposal in subsection $\mathrm{A}$ is its computational ease. Nevertheless, the worth index computation of this section may singularly be simplified if the computational propositions in [21] are used instead of applying Zadeh's extension principle. Indeed, as we are dealing with piecewise linear distributions, and with a piecewise linear aggregation operator, the aggregated possibility distribution is also piecewise linear. Moreover, the Choquet integral needs only to be calculated at the intersection points of the ascending and descending parts of the input possibility distributions [21]. 


\section{The cost/benefit assessment}

The decision maker can now choose the criteria to be improved starting from $x^{0}$ : in the two approaches, he can only compare $w_{C_{\mu}}^{*}\left(x_{0}, I\right)$ for all $I \subseteq N$. For sake of simplicity, the direct costs of improvements has not been introduced directly in the model (see [10] and [11]). As previously mentioned, we merely consider that the fewer criteria to be improved, the lower the direct cost of the increase. Because, $w_{C_{\mu}}^{*}\left(x_{0}, N\right)=\max _{I \subseteq N}\left\{w_{C_{\mu}}^{*}\left(x_{0}, I\right)\right\}$ when direct cost is not explicitly taken into account in the worth index model, the decision maker can choose $I^{*}$ such that for two small real numbers $\epsilon$ and $\eta,\left|w_{C_{\mu}}^{*}\left(x_{0}, N\right)-w_{C_{\mu}}^{*}\left(x_{0}, I\right)\right|<\epsilon$ whereas $\frac{\left|I^{*}\right|}{|N|}<\eta$ : improving criteria in $I^{*}$ has the potential to produce a benefit close to the maximal expectation whereas there are much fewer criteria to be improved in $I^{*}$.

\section{ApPLICATION}

\section{A. Scope and Available Data of the Application}

The robotic challenge Robafis is organized annually by the French association of Systems Engineering AFIS to promote Systems Engineering practice in engineers's schools. The scope of the challenge is for instance in Robafis 2013 (www.afis.fr) to build an autonomous mobile robot able to compete with other robots and using some provided and imposed materials. Each robot is limited $30 \mathrm{~cm}^{3}$ and has to achieve the following mission as quickly as possible: to grasp and transport some various coloured spheres between several stock devices spread over a plan playground. Some dark lines are drawn on the ground to guide the robot between stock devices.

Given an already built robot prototype, the question is then: on which criteria the designer team has to focus in order to improve as much as possible the prototype and to win the challenge. Four criteria are considered to evaluate any robot configuration: the material cost of the robot (CR1), the robot speed capacity (CR2), the reliability of the robot (CR3), and its maintainability (CR4). We have chosen the Choquet integral as the aggregation operator to merge theses four criteria. An interactive and iterative procedure has been carried out to capture the DM preferences using the extension of the indirect identification method MACBETH proposed in [8] [14]. The result is the fuzzy measure associated to the Choquet integral given in Table 1.

\begin{tabular}{|c|c|c|c|}
\hline$I$ & $\mu(I)$ & $I$ & $\mu(I)$ \\
\hline$\emptyset$ & 0 & $\{2\}$ & 0 \\
\hline$\{1\}$ & 0 & $\{2,4\}$ & 0.57 \\
\hline$\{1,4\}$ & 0 & $\{1,2\}$ & 0.14 \\
\hline$\{3\}$ & 0 & $\{1,2,4\}$ & 0.71 \\
\hline$\{4\}$ & 0 & $\{2,3\}$ & 0.71 \\
\hline$\{1,3\}$ & 0.28 & $\{2,3,4\}$ & 0.71 \\
\hline$\{3,4\}$ & 0 & $\{1,2,3\}$ & 0.86 \\
\hline$\{1,3,4\}$ & 0.43 & $\{1,2,3,4\}$ & 1 \\
\hline
\end{tabular}

TABLE I: The obtained fuzzy measure

We assume that experts are able to provide the possibility distributions over the value range of performance according to the criteria. The possibility distributions associated to the four criteria are given in Figure 1. The performance of the prototype to be improved has been measured equal to $x^{0}=$ $(0.5,0.1,0.2,0.5)$.

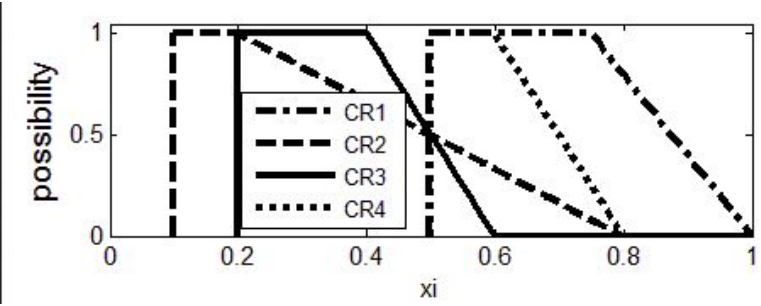

Fig. 1: Possibility distributions of attributes performances

\section{B. Computing the possibilistic worth index}

We seek which subsets of criteria $I \subset N$ are the most promising to improve as much as possible the overall performance of the prototype. For that, let us apply the two proposed approaches to compute the worth index associated to any nonempty subset of criteria $I$. For the sake of comparison, the values of the worth index proposed in [10] where all the improvements are considered as equiprobable are also computed. All the results are given in Table II. IV-A (resp. IVB) is the values of the worth index obtained using the approach presented in subsection IV-A (resp. subsection IV-B), while (ref. [10]) is the one obtained using the formula (2).

\begin{tabular}{|c|c|c|c|}
\hline$I$ & \multicolumn{3}{|c|}{$w_{C_{\mu}}\left(x^{0}, I\right)$} \\
& IV-A & IV-B & ref. [10] \\
\hline$\{1\}$ & 0 & 0 & 0 \\
\hline$\{2\}$ & {$[0 ; 0.2700]$} & {$[0 ; 0.2168]$} & 0.2077 \\
\hline$\{1,2\}$ & {$[0 ; 0.2700]$} & {$[0 ; 0.2273]$} & 0.2271 \\
\hline$\{3\}$ & {$[0 ; 0.1290]$} & {$[0 ; 0.0215]$} & 0.1048 \\
\hline$\{1,3\}$ & {$[0 ; 0.1290]$} & {$[0 ; 0.1215]$} & 0.1486 \\
\hline$\{2,3\}$ & {$[0 ; 0.3570]$} & {$[0 ; 0.3213]$} & 0.3808 \\
\hline$\{1,2,3\}$ & {$[0 ; 0.3570]$} & {$[0 ; 0.3320]$} & 0.4065 \\
\hline$\{4\}$ & 0 & 0 & 0 \\
\hline$\{1,4\}$ & 0 & 0 & 0 \\
\hline$\{2,4\}$ & {$[0 ; 0.2700]$} & {$[0 ; 0.2595]$} & 0.2868 \\
\hline$\{1,2,4\}$ & {$[0 ; 0.2700]$} & {$[0 ; 0.2700]$} & 0.3063 \\
\hline$\{3,4\}$ & {$[0 ; 0.1290]$} & {$[0 ; 0.1075]$} & 0.1048 \\
\hline$\{1,3,4\}$ & {$[0 ; 0.1290]$} & {$[0 ; 0.1290]$} & 0.1720 \\
\hline$\{2,3,4\}$ & {$[0 ; 0.3570]$} & {$[0 ; 0.3498]$} & 0.3808 \\
\hline
\end{tabular}

TABLE II: The three different worth index values

\section{Results Analysis}

According to Table II we can note that:

- The upper bound of the worth index $w_{C_{\mu}}\left(x^{0},.\right)$ is monotonous non decreasing for the partial inclusion with respect to $I$. So, the best expected improvement is naturally obtained for $I=\{1,2,3,4\}$.

- The worth of improving the robot on criteria : $\{2,3\}$, $\{2,3,4\}$ is almost the same but the direct cost of improving $\{2,3\}$ is lower than the one of $\{2,3,4\}$ (see the direct cost assumption in subsection IV-C). Thus $\{2,3\}$ (speed capacity and reliability) are the criteria to be improved to expect the most satisfying performance at the least direct cost. 
- For any $I \subset N$, the worth index based on the moments of the possibility distributions (IV-A) includes the worth index based on uncertainty propagation (IV-B).

In this illustrative case study, discrepancies between worth indexes in Table II are not so demonstrative because of the simplicity of the example. The values of the worth index based on the moments of the possibility distributions (IV-A) are necessarily more optimistic that the ones based on uncertainty propagation (IV-B) since $C_{\mu}\left(E^{*}\left(\pi_{1}\right), \ldots, E^{*}\left(\pi_{n}\right)\right) \geq$ $E^{*}\left(C_{\mu}\left(\pi_{1}, \ldots \pi_{n}\right)\right.$ always holds (see [18] for more details). In the case of intersections $E^{*}\left(C_{\mu}\left(\pi_{1}, \ldots \pi_{n}\right)\right.$ can significantly differ from $C_{\mu}\left(E^{*}\left(\pi_{1}\right), \ldots, E^{*}\left(\pi_{n}\right)\right)$ which provides less constrained expectations. In our illustrative example, it happens for example for subsets $\{2\},\{1,2\}, \ldots$ etc. (see Table II). The discrepancy is more significant when comparing our worth index with the one in [10] where all the improvements are assumed to be equiprobable whatever their magnitude. It is easily understandable because the expert knowledge tends to claim that the greater the improvement, the less it is possible.

Finally note that the worth indexes have been computed for all the criteria $I \subset N$ for a single starting point $x^{0}$ that represents the initial configuration (or prototype in our example) from which the improvement is envisaged. In our future works, a set of potential candidate initial configurations will be considered in the preliminary design stage. For all of them, the worth indexes will be computed and compared: the selected initial configuration will be the one that has the greatest potential for improving the overall performance.

\section{CONCLUSION}

Improving product properties in today's competitive market is mandatory but presents important costs and risks. Supporting DM's in their decision making is thus a strategic challenge. However, because of insufficient knowledge about the performances that can be expected from improvement decisions, the only available information are often incomplete and imprecise, making early engineering decisions risky. Thus, identifying the criteria to be profitably improved while not exceeding the scope of one's own skills and competence appears to be very important for helping decision makers to focus efficiently on pertinent targets. That's why we propose here two different extensions of the worth index (the purpose of which is to identify the most promising criteria) in a possibility theory framework. The case study of the reengineering of an autonomous robot illustrates the proposed approach. In further works we are going to extend these research results for prognostic purposes where the possibility distributions related to the expected improvements may be affected by disturbances or faults.

\section{REFERENCES}

[1] A. Baykasoğlu, A. Öztaş, and E. Özbay, "Prediction and multi-objective optimization of high-strength concrete parameters via soft computing approaches," Expert Systems with Applications, vol. 36, no. 3, pp. 61456155,2009
[2] K. Ng, "A critical analysis of current engineering design methodologies from a decision making perspective," in Proceedings of the 2nd International Virtual Conf. on Innovative Production Machines and Systems (I* PROMS 2006), 2011, pp. 369-374.

[3] V. Moulianitis, N. Aspragathos, and A. Dentsoras, "A model for concept evaluation in design - an application to mechatronics design of robot grippers," Mechatronics, vol. 14, no. 6, pp. 599-622, 2004.

[4] J. R. Hauser and D. Clausing, "The house of quality," Harvard business review, vol. 66, no. 3, 1988

[5] Z. Babic and N. Plazibat, "Ranking of enterprises based on multicriterial analysis," International journal of production economics, vol. 56, pp. 2935, 1998.

[6] L. Berrah, G. Mauris, J. Montmain, and V. Clivillé, "Efficacy and efficiency indexes for a multi-criteria industrial performance synthesized by choquet integral aggregation," International Journal of Computer Integrated Manufacturing, vol. 21, no. 4, pp. 415-425, 2008.

[7] J.-L. Marichal, "An axiomatic approach of the discrete choquet integral as a tool to aggregate interacting criteria," IEEE Transactions on Fuzzy Systems, vol. 8, no. 6, pp. 800-807, 2000.

[8] C. Labreuche and M. Grabisch, "The choquet integral for the aggregation of interval scales in multicriteria decision making," Fuzzy Sets and Systems, vol. 137, no. 1, pp. 11-26, 2003.

[9] M. Grabisch and C. Labreuche, "How to improve acts: an alternative representation of the importance of criteria in mcdm," International Journal of Uncertainty, Fuzziness and Knowledge-Based Systems, vol. 9, no. 02, pp. 145-157, 2001.

[10] C. Labreuche, "Determination of the criteria to be improved first in order to improve as much as possible the overall evaluation," in Int. Conf. on Information Processing and Management of Uncertainty in Knowledge-Based Systems (IPMU), Perugia (ITA), 2004, pp. 609-616.

[11] J. Montmain, C. Labreuche, A. Imoussaten, and F. Trousset, "Multicriteria improvement of complex systems," Information Sciences, vol. 291, pp. 61-84, 2015

[12] A. Imoussaten, B. Duthil, F. Trousset, and J. Montmain, "Identifying priority lines of improvement. application to tourism data,." LFA 2016, La Rochelle, France, 2016.

[13] D. Krantz, D. Luce, P. Suppes, and A. Tversky, Foundations of measurement, Vol. I: Additive and polynomial representations, 1971.

[14] M. Grabisch, I. Kojadinovic, and P. Meyer, "A review of methods for capacity identification in choquet integral based multi-attribute utility theory: Applications of the kappalab r package," European journal of operational research, vol. 186, no. 2, pp. 766-785, 2008.

[15] D. Bouyssou and M. Pirlot, "Conjoint measurement tools for mcdm," in Multiple Criteria Decision Analysis: State of the Art Surveys. Springer, 2005, pp. 73-112.

[16] D. Dubois and H. Prade, "Possibilistic theory: an approach to computerized processing of uncertainty new york," 1988.

[17] _ "The mean value of a fuzzy number," Fuzzy sets and systems, vol. 24, no. 3, pp. 279-300, 1987.

[18] A. Denguir-Rekik, J. Montmain, and G. Mauris, "A possibilistic-valued multi-criteria decision-making support for marketing activities in ecommerce: Feedback based diagnosis system," European Journal of Operational Research, vol. 195, no. 3, pp. 876-888, 2009.

[19] A. Imoussaten, J. Montmain, and G. Mauris, "A multicriteria decision support system using a possibility representation for managing inconsistent assessments of experts involved in emergency situations," International Journal of Intelligent Systems, vol. 29, no. 1, pp. 50-83, 2014.

[20] L. A. Zadeh, "Fuzzy sets," Information and control, vol. 8, no. 3, pp. 338-353, 1965.

[21] M. Grabisch, H. T. Nguyen, and E. A. Walker, Fundamentals of uncertainty calculi with applications to fuzzy inference. Springer Science \& Business Media, 2013, vol. 30.

[22] G. Mauris, "A review of relationships between possibility and probability representations of uncertainty in measurement," IEEE Transactions on Instrumentation and Measurement, vol. 62, no. 3, pp. 622-632, 2013. 